\title{
Click or Tap Here to Enter Text
}

National Cancer Institute

\section{Source}

National Cancer Institute. Click or Tap Here to Enter Text. NCI Thesaurus. Code C158624.

A directive to click or tap to enter text. 